\title{
Wideband Circularly Polarized Antenna with Enhanced Bandwidth and Conical Radiation Pattern
}

\author{
Sandeep Kumar Modi \\ Department of ECE, MANIT
}

Bhopal

\author{
Hirdesh Chack \\ Department of ECE, MANIT \\ Bhopal
}

\author{
Ajay Somkuwar, PhD \\ Profesor,Department of ECE \\ MANIT,Bhopal
}

\begin{abstract}
In this paper a new technique is used to enhance the band width with standard gain using HFSS software. The radiation pattern has been investigated in $\mathrm{C}$ and $\mathrm{X}$ band. The design of wideband antenna is very challenging when there is a tradeoff in between gain and bandwidth. The reason to simulate this antenna in HFSS is because of its easy simulation and optimization of the various dimension of antenna. The design consists of a feeding probe and rectangular parallelepiped of different parasitic dielectric constant to surround the feeding probe.In this paper a new design of antenna is studied .The proposed structure is very simple and wide band. The wideband characteristics are observed in the frequency range of $5.18 \mathrm{GHz}$ to $8.71 \mathrm{GHz}$. The above design has $10-\mathrm{dB}$ impedance band width of more than $65 \%$. Gain obtained in this design is average. But this gain is distributed over all band width. Maximum gain is at $7.78 \mathrm{GHz}$ and equal to 6.34 $\mathrm{dB}$
\end{abstract}

\section{General Terms}

Bandwidth improvement, circular radiation pattern,

\section{Keywords}

Antenna feeds, Electromagnetic propagation, Dielectric constant, Wideband antenna, Circular polarization

\section{INTRODUCTION}

Antenna is basically an electrical device which can be used as a transmitter or receiver or can say it converts electrical power into radio waves or vice versa. With the new technology in demand and rapid development in mobile communication the only thing that we have to pay is band width. Now a days in any communication system main challenge is to use the available band width more efficiently. The above antenna is designed for very high frequency band and it is circularly polarized, so can be used extensively used in moving vehicles like ship and satellite. Due to Omni directional property they can be used as fixed mobile station for larger signal coverage.

In general a linearly polarized wave is easy to get as compare to circularly wave polarized wave, but circularly wave polarized have its own advantages over linearly polarized wave. So a polarizer is used here to do so. In this paper the same is obtained by integrating the feeding probe with a polarizer composed of dielectric parallelepiped. The feeding probe is situated at the center of circular ground plane and dielectric parallelepiped is situated at the circumference of ground plane. Axial ration is an important parameter to define how much the wave is circularly polarized. Ideally for a circularly polarized antenna it should be ' 1 ' or can say ' 0 ' $\mathrm{dB}$. The variation of axial ration from its ideal value is due to the presence of metallic structure on which antenna is mounted or the element through which the feeding probe is surrounded. The characteristics of antenna like band width, gain, axial ratio and radiation pattern is studied and simulated using HFSS software.

\section{ANTENNA DESIGN AND THEORY}

The basic principle of antenna is to transmit or receive the information through radio waves. For any wireless communication system, we can't even think of a system without antenna. They are widely used in radio broadcasting, broadcasting TV, radar, mobile communication, satellite communication, Bluetooth, wireless microphones, GPS etc. Depending upon requirement it can be designed to transmit or receive in all direction or in particular direction.

Polarization of antenna plays significant role in field orientation of radio waves. Linear polarized antenna is very cheap, easy to design and work well enough in daily application and in daily application cross polarization does not occur, but while flying FPV, we constantly change the orientation of antenna. In every turn or roll of aircraft the polarization is shifted and so data can be lost, so circular polarization comes into picture

Instead of transmitting in just one plane, a circular polarized antenna transmits data in both planes at once with a 90 degree phase. So here we produce linear polarized wave and then with the help of wave polarizer we get circular polarized wave. For this

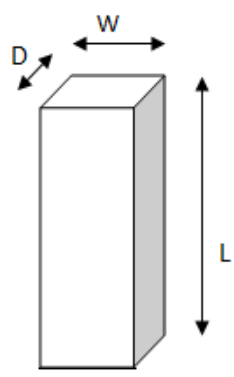

Figure 1

we are using parallelepiped which is made of different dielectrics. In Fig. 1 a parallelepiped has shown that consists of dielectric slab with length $\mathrm{L}$ and depth $\mathrm{D}$.

The wave polarizer works on the principle of breaking the electric field component into two axis. $E_{x}$ and $E_{y}$ are two electric field component travelling with different velocity causing them to have different phase componenet. Exact circular polarizer wave can be obtained when both $\mathrm{E}_{\mathrm{x}}$ and $\mathrm{E}_{\mathrm{y}}$ have equal magnitude. Fig. 2 shows a polarizer. Here the linear polarized wave is assumed to coming through $\mathrm{z}$ axis and when it comes to polarizer, breaks into to componenet. 


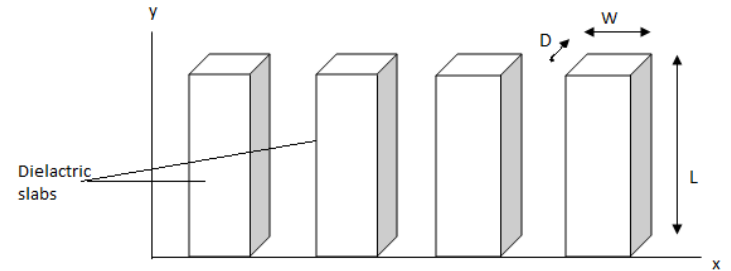

Figure 2

In this paper the antenna source is made of coaxial type probe, which simultaneously work as a monopole antenna, as it is in vertical direction so it will have dominantly vertically polarized wave. Dielectric slab of different dielectric constant works as a polarizer. The used dielectric slab is not perfect parallelepipeds, but some part of it is removed from it to match the polarization perfectly and to get the proposed bandwidth. The removed part for the entire slab is same. Fig 3 shows the configuration of proposed structure. Fig 4 shows the top views of the proposed structure. The parallelepipeds are distributed not exactly along the circumference of the ground plane but near to the circumference. They are situated at a distance of $\mathrm{R}^{\prime}=17 \mathrm{~mm}$ from the feeding probe. The angular interval between each parallelepipeds is defined by $360^{\circ} / \mathrm{N}$. Here $\mathrm{N}=4$, so angular interval $=90^{\circ}$. According to Fig. 3, the proposed probe has a length 1 and diameter $d$. The radius of ground plane is $R$.

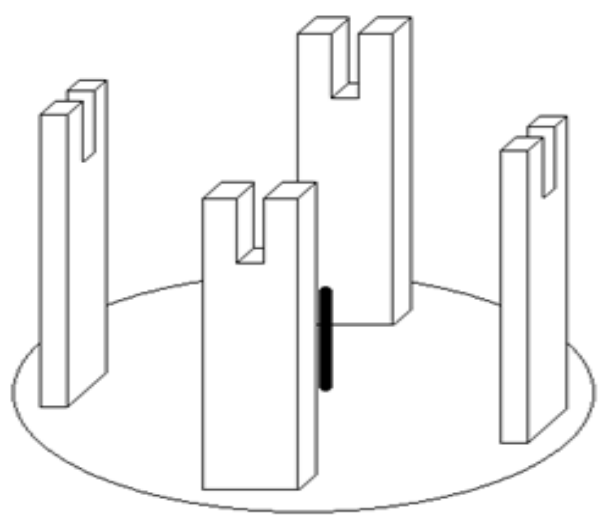

Figure 3

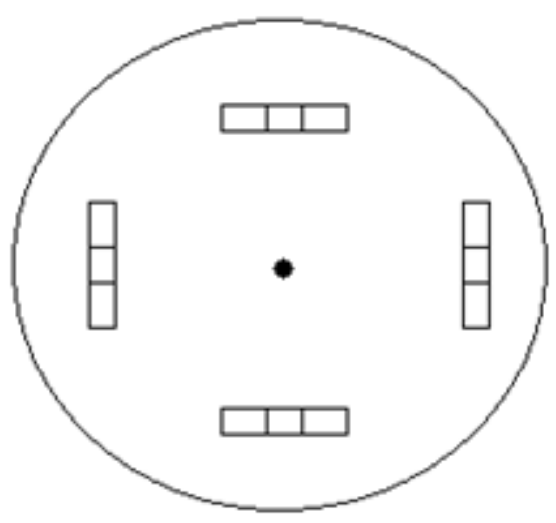

Figure 4

In our proposed antenna I have used co- axial feeding technique. The outer surface is connected to the substrate i.e. ground plane, which is taken as ground plane. The input signal is given to the input surface, it means the core of coaxial cable. The main advantage of this type of feeding is that, feed can be given to any desired location in the substrate to match the input impedance. Also the modeling of such a narrow hole to drill into the ground and get a planer surface over it is another major advantage. Here in this antenna the feeding probe is situated over the center of the ground plane. Major advantage of using this technique is that, the feed can be placed at any place on the substrate.

\section{SIMULATED RESULTS}

A new type of circularly polarized antenna is investigated and designed and tested. The parameter of the proposed structure is as follows. $\varepsilon_{\mathrm{r} 1}=20, \varepsilon_{\mathrm{r} 2}=2.96, \varepsilon_{\mathrm{r} 3}=20, \varepsilon_{\mathrm{r} 4}=2.96, \mathrm{~N}=4$, $\mathrm{L}=62 \mathrm{~mm}, \mathrm{~W}=16 \mathrm{~mm}, \mathrm{D}=3 \mathrm{~mm}, \mathrm{R}=36.5 \mathrm{~mm}, \mathrm{R}=17 \mathrm{~mm}$, $l_{p}=13.5 \mathrm{~mm}, \mathrm{r}=0.63 \mathrm{~mm}$.

Although the proposed structure has a certain height, the antenna is not heavy; it is because cross section area of used parallelepipeds is small. So this antenna can be used widely in many communication systems. Although the proposed structure has a certain height, the antenna is not heavy; it is because cross section area of used parallelepipeds is small. So this antenna can be used widely in many communication systems.

From the figure 5, it is very clear that we are getting two resonance frequencies, each for feeding probe and the parallelepiped combination. The resonance frequency is a function of the dielectric constant of parallelepiped. The lower resonance frequency is due to the probe and the higher resonance frequency is due to parallelepiped

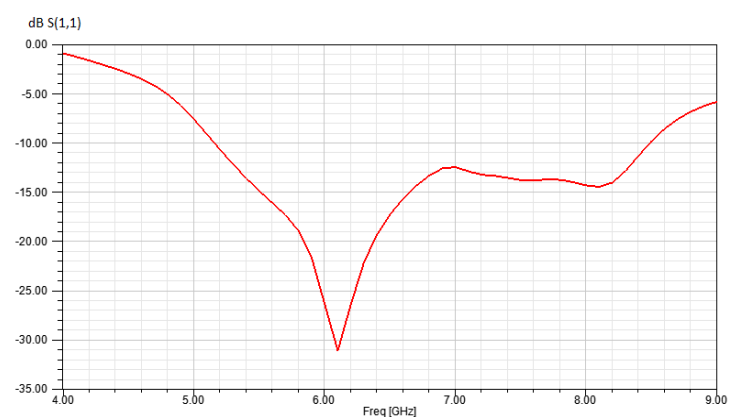

Figure 5 Simulated result showing two resonance frequency

This can be verified by taking the value of each parallelepiped is unity (i.e. $\varepsilon_{\mathrm{r}}=1$ ) this means that the effect of dielectric parallelepiped vanishes, and the resonance frequency is only due to feeding probe. In this case from graph it can be seen that only resonance frequency is $6.25 \mathrm{GHz}$. As we increases the value of dielectric constant, than the effect of parallelepiped comes into picture. And next resonance frequency is obtained. From the figure 6 it is also very clear that as the value of dielectric constant increased, than the lower resonance frequency due to feeding probe shifts to lower value, but for value $\varepsilon=10 \& 15$, we get multiple resonance frequency which occur at different frequency but only two of them are strongest among them. But the second resonance frequency due to parallelepiped doesn't follow regularity; first it decreases and then increased for different frequency and then finally decreased 


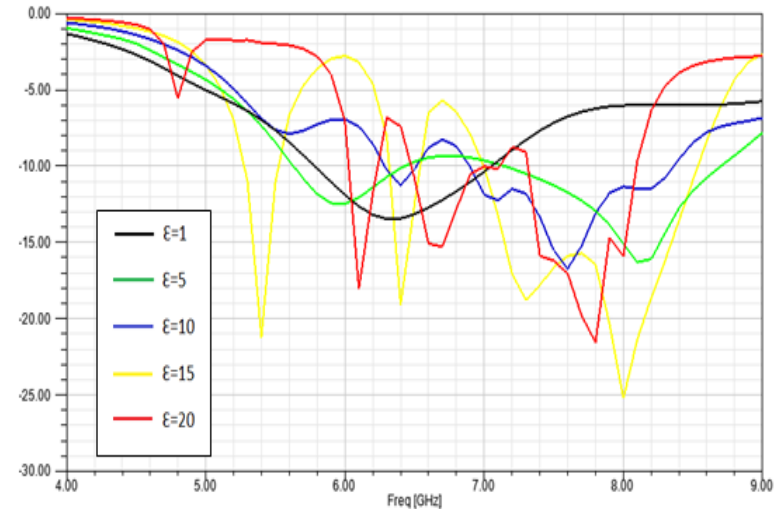

Figure 6 Simulated reflection constant with different dielectric constant

If we fix the dielectric constant of two parallelepipeds is 2.94 , and then vary the dielectric constant of the remaining two, then we get different characteristics. The resonance frequency due to feeding probe gets stronger and resonance frequency due to parallelepiped shifts to a lower value. Here obtained graph shows certain regularity. Here when we take the initial value or the lowest value of dielectric constant of two variable slabs is ' 5 ' than it has the maximum bandwidth, but at frequency range 7.1 to $7.9 \mathrm{GHz}$, graph goes above $-10 \mathrm{~dB}$ line. It shows that this frequency band is not useful. For dielectric constant of slab equal to 10 , we get a frequency range from 5.25 to $8.51 \mathrm{GHz}$. Here this complete frequency range is below $-10 \mathrm{~dB}$. Also the lower resonance frequency range shifted to a lower frequency and the higher resonance frequency shifts to slightly higher frequency then previous value of dielectric constant of slab. For dielectric constant of 15 for varying slab, lower resonance frequency shifts to higher frequency and the higher resonance frequency shifts to lower value. Due to this overall bandwidth reduces. For final value of 20 , we get the used frequency range from 5.15 to $8.45 \mathrm{GHz}$. If we compare the plot at the value of dielectric constantof slab at 10 and 20 , then we can see that the overall used bandwidth is approximate same, but other parameter like gain and axial ratio at dielectric constant of 10 is bad as compare to 20. So we choose the dielectric constant 20 for most optimize value. This is shown in figure 7 .

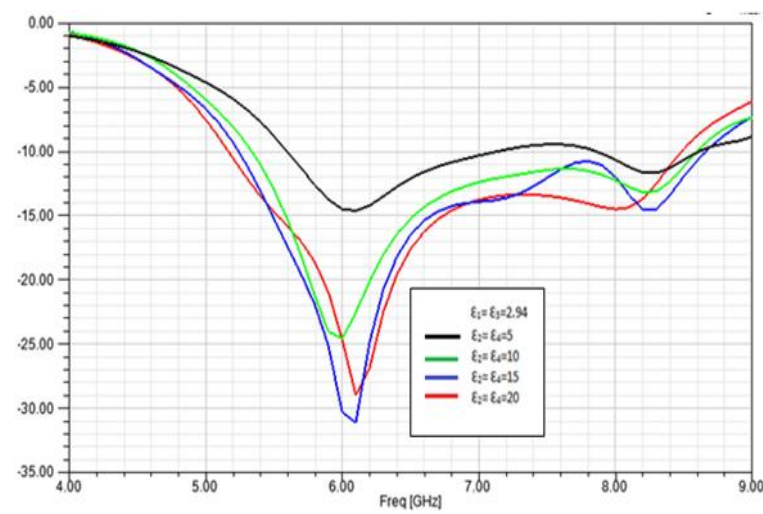

Figure 7 Simulated reflection constant keeping reflection coefficients of two slabs constant

Resonance frequency is also the function of distance between feeding probe and the dielectric slab. With increasing the distance from feeding probe to dielectric slab the lower resonance frequency due to feeding probe, continuously increases and the higher resonance frequency due to dielectric slab, continuously decreases. From the graph it is very clear that when distance between feeding probe and the dielectric slab is lowest i.e. $\mathrm{R}^{\prime}=12 \mathrm{~mm}$ then useful bandwidth is very low. It is worst among all. With continuously increasing the distance used, overall bandwidth also increases. With increasing distance the resonance frequency also getting stronger. For all value accept $\mathrm{R}^{\prime}=18$, there is certain frequency range between resonance frequency, that is above $10 \mathrm{~dB}$. This is not useful for practical purpose. For $\mathrm{R}^{\prime}=18$ $\mathrm{mm}$, we are getting maximum frequency range and between resonance frequency all frequency is useful. At $R^{\prime}=18 \mathrm{~mm}$, we are getting maximum bandwidth. This is shown in Figure.8

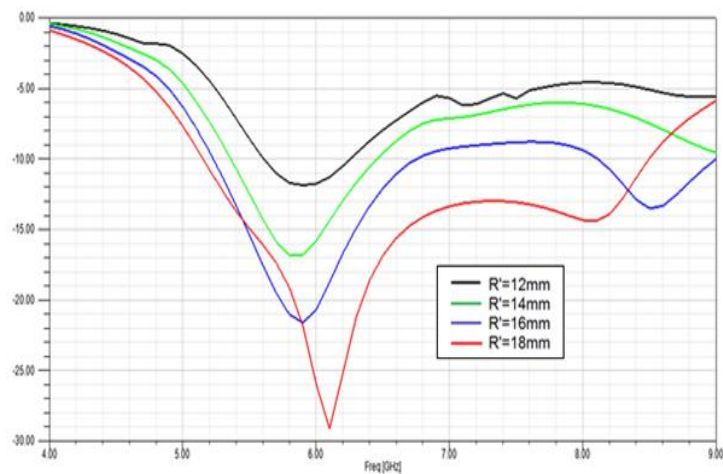

\section{Figure 8 Simulated Reflection co-efficient with varying distance between feeding probe and slab}

The bandwidth and resonance frequency is very strong function of the height of the feeding probe. up to a height of $9.5 \mathrm{~mm}$ of feeding probe we don't get any useful bandwidth. After a height of $10 \mathrm{~mm}$ we get some useful bandwidth, but the resonance bandwidth is not strong. Further when we increases the height of the probe, the lower resonance frequency due to the feeding probe comes into picture and shifts to lower frequency for further increased in height. The higher resonance frequency due to parallelepiped shifts to higher resonance frequency with increasing height. For the lowest value of height probe $1_{p}=10 \mathrm{~mm}$, resonance frequency is not very strong and used frequency is very less. For $l_{p}=$ $11 \mathrm{~mm}$, the obtained frequency range is from 6.26 to $8.9 \mathrm{GHz}$. For $l_{p}=12 \mathrm{~mm}$, used frequency is from 5.7 to $8.62 \mathrm{GHz}$. Maximum useful bandwidth is obtained when we take the height of feeding probe $l_{p}=12.8 \mathrm{~mm}$. At this value used frequency lie between 5.1 to $8.45 \mathrm{GHz}$. This is shown in figure 9.

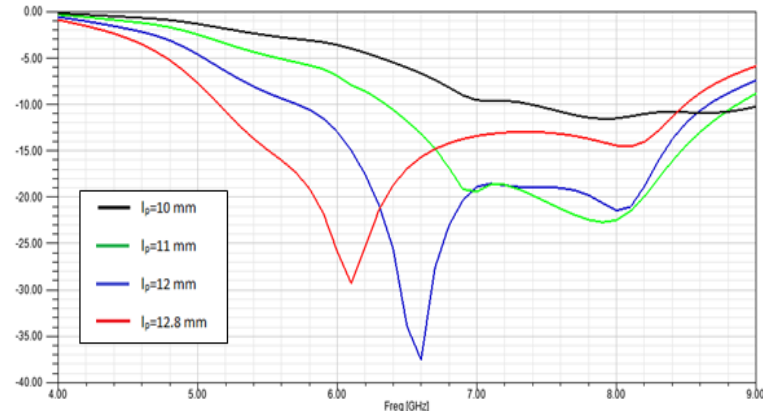

\section{Figure 8 Simulated Reflection co-efficient with different} hight of feeding probe

Bandwidth of the antenna is defined as the frequency range over which antenna works satisfactory. Our antenna is designed to works mainly on the frequency range from 4 to 9 $\mathrm{GHz}$. It means basically it covers the entire $\mathrm{C}$ band and some part of X band. Also our satellite communication works in this 
band of frequency. So this designed antenna has lots of bandwidth. Here used bandwidth is from 5.14 to $8.50 \mathrm{GHz}$. This is because only this frequency range lies below the -10 $\mathrm{dB}$ line. This line is used as standard line, as it defines that loss is minimum in this frequency range. From graph we can see that total used bandwidth is approximately $67.2 \%$ of all available bandwidth. This is shown in figure 9 .

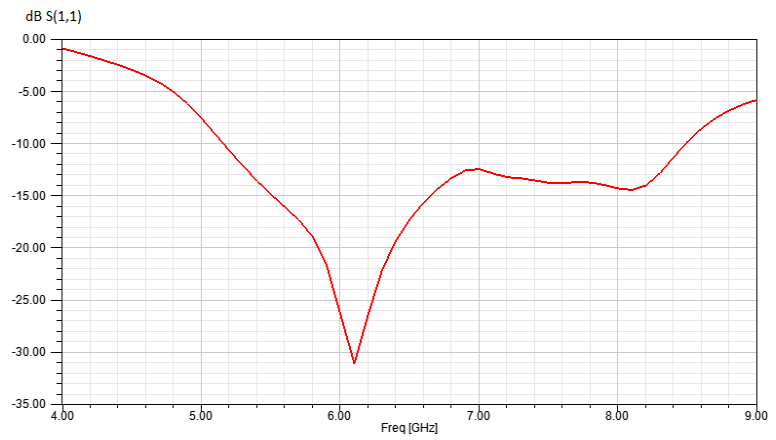

Figure 9 Final bandwidth of our proposed antenna

Radiation pattern of antenna shows the plot of power radiated as a function of direction away from antenna. The power radiated by antenna is defined as a function of some spherical co-ordinates. This is done because ideal antenna is supposed to radiate the power in all symmetrical direction. But practically an antenna can't be a complete Omni directional, but have a certain maximum power in certain direction and vice versa. As radiation pattern is a 3-D structure, but to draw it in paper to understand it, we use a 2-D view. Here radiation pattern of our antenna drown. This plot is drawn for a particular frequency of $5 \mathrm{GHz}$. This is shown in figure 10 . From the plot it is very clear that the radiation plot is not symmetrical in all direction. In some direction it is maximum and some direction it is minimum. At $\theta=0^{\circ}$ and $180^{\circ}$ it has minimum power flow. At $\theta=80^{\circ}$ it has maximum power flow in that direction.

Where $\theta$ shows the maximum electric field in that direction and

$\Phi$ shows the maximum electric field in that direction.

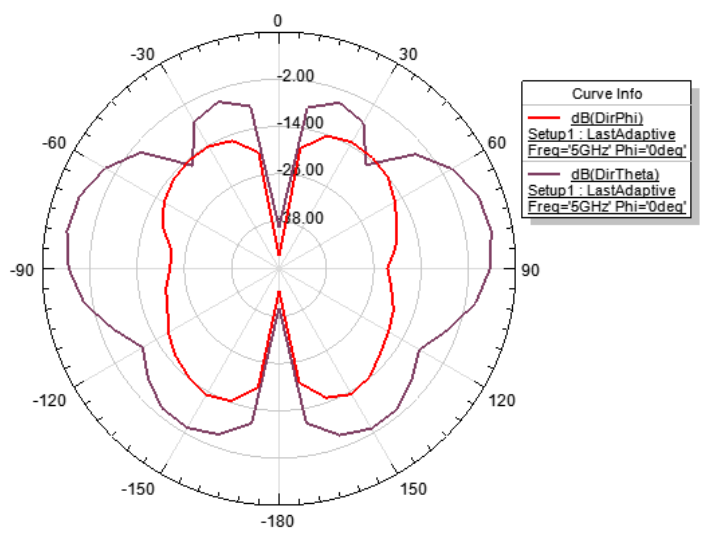

Figure 10 Radiation Pattern of our proposed antenna

Axial ratio of the antenna is also calculated here. It is a very important parameter for antenna designing. For ideal circular polarized antenna the axial ratio will be $0 \mathrm{~dB}$. It is the ratio of orthogonal electric field component. Deviation from its ideal value of axial ratio, it shows how much antenna is circularly polarized. The axial ratio performance of antenna is not very good. Up to some frequency range performance of antenna is good, but at some frequency like at $5.89 \mathrm{GHz}$ and at 7.34 $\mathrm{GHz}$, its axial ratio performance is bad. It shows that at that frequency range the antenna wouldn't give desire polarization. To plot axial ratio of antenna, we need to fix some value. Here it is plotted at frequency of $5 \mathrm{GHz}$. This is shown in figure 11. From radiation pattern plot drown below, it is very clear that the radiation is maximum at the angle $\theta=80^{\circ}$ and $\Phi=0^{\circ}$.

Where $\theta$ and $\Phi$ are the direction in which electric field is maximum.

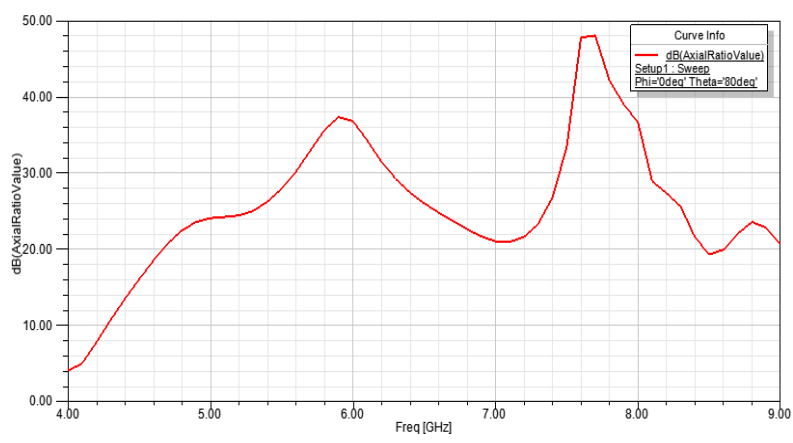

Figure 11 Axial ratio of our proposed antenna

Gain of the antenna defines the how much energy it can transfer energy in a particular direction. For an isotropic antenna it is $0 \mathrm{~dB}$. For batter performance its value should be high. Here antenna gain in terms of $\mathrm{dB}$ is calculated and shown in figure 12.Antenna gain with a function of frequency is plotted and verified. Here we have taken frequency range from 4 to $9 \mathrm{GHz}$, and gain is plotted for that interval. Obtained antenna gain is maximum at $7.78 \mathrm{GHz}$. Its maximum value is $6.34 \mathrm{~dB}$. Such gain is sufficient for this type of antenna. Here obtained antenna gain is distributed over entire bandwidth. So we can get high gain over all frequency range.

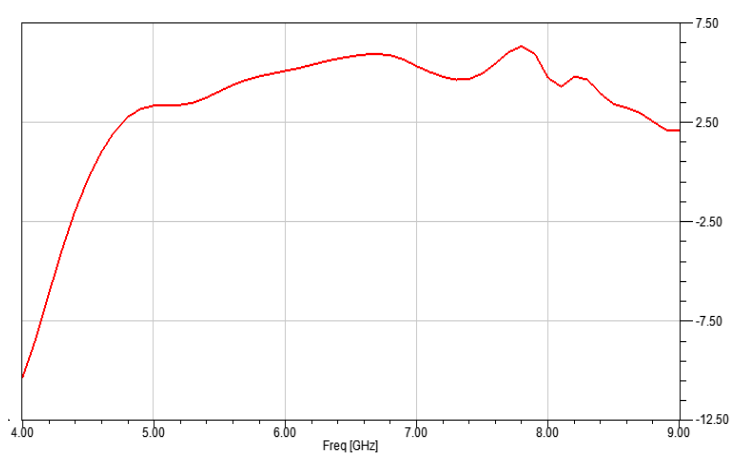

Figure 12 Gain of our proposed antenna

Radiated field of antenna is very important parameter in antenna design. It shows the distribution of electric field with the function of entire available frequency range.

The maximum left hand circularly polarized component $=$ $\frac{1}{\sqrt{2}}\left(E_{\theta}-j E_{\Phi}\right)$

The maximum left hand circularly polarized component $=$ $\frac{1}{\sqrt{2}}\left(\mathrm{E}_{\theta}+\mathrm{j} \mathrm{E}_{\Phi}\right)$

Where $\theta$ and $\Phi$ are the direction in which electric field is maximum.

This field is shown in figure 13. 


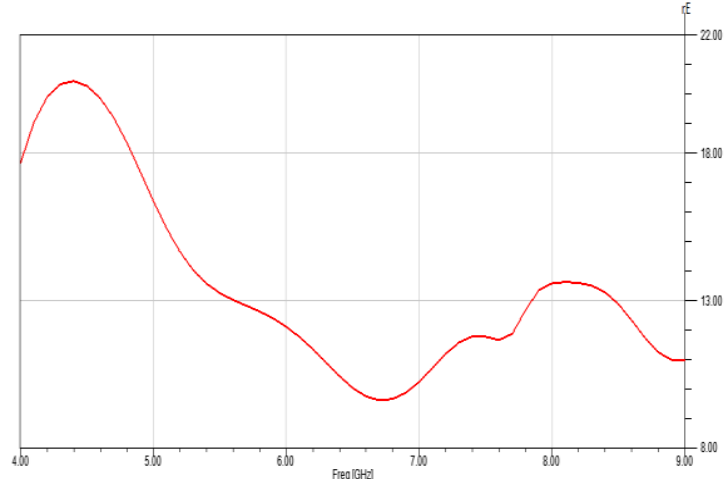

Figure 13 Radiated electric field of our proposed antenna

\section{CONCLUSION}

In this thesis we have discussed a method to design a new type of antenna, which is compatible in size, very efficient and covers a long bandwidth in $\mathrm{C}$ band and some part of $\mathrm{X}$ band. The antenna that we have designed has major advantage of efficient bandwidth of entire frequency band. Here the available bandwidth is from 4 to $9 \mathrm{GHz}$. With conventional method we can use only $30 \%$ of available bandwidth. But with new technique proposed here, we can enhance it up to 68 $\%$. Gain obtained in this design is average.But this gain is distributed over all band width. Maximum gain is at $7.78 \mathrm{GHz}$ and equal to $6.34 \mathrm{~dB}$. The axial ratio that we are getting in this technique is not so good.

\section{REFERENCES}

[1] J.C.Maxwell, "A Treatise on Electricity and Magnetism" Vol.1-2, Oxford, 1873.

[2] George W. Hanson, IEEE transactions on antennas and propagation,vol.41, no.12, December1993

[3] Karen Cummings, Priscilla Laws, EdwadRedishand Patrick Cooney, "Understanding Physics" John Wiley \& Sons (Asia) Pvt.Ltd. Wiley India edition.

[4] DRDO Science Spectrum, March 2009, pp 66-78

[5] C. A. Balanis; Antenna Theory: Analysis and Design, John Wiley \& Sons (Asia) Pvt.Ltd., Singapore.
[6] Pekka M. T. Ikonen, Student Member, IEEE, Pekka Alitalo, Sergei A. Tretyakov, Senior Member, IEEE“On Impedance Bandwidth of Resonant Patch Antennas Implemented Using Structures with Engineered Dispersion"

[7] R.V.S. Ram Krishna, Raj Kumar "Design ofultra wideband trapezoidal shape slot antenna with circular polarization"Volume 67, Issue 12, December 2013 , Pages 1038-1047

[8] K. L. Lau and K. M. Luk, "A wideband circularly polarized conical beam patch antenna,"IEEE Trans. Antennas Propag., vol. AP-54, pp.1591-1594, May 2006.

[9] Yong-Mei Pan, Member, IEEE, and Kwok Wa Leung, "Wideband Circularly Polarized Dielectric Bird-Nest Antenna with Conical Radiation Pattern" IEEE transactions on antennas and propagation, vol. 61 , no. 2 , february 2013.

[10] J. D. Kraus, Antennas, 2nd ed. New York: McGraw-Hill, 1988.

[11] H. S. Kirschbaum and L. Chen, "A method of producing broadband circular polarization employing an anisotropic dielectric," IRE Trans.Microwave Theory Tech., vol. 5, no. 3, pp. 199-203, 1957.

[12] H. Kawakami, G. Sato, and R. Wakabayashi, "Research on circularly polarized conical-beam antennas," IEEE Antennas Mag., vol. 39, pp.27-39, Jun. 1997.

[13] H. Nakano, K. Fujimori, and J. Yamauchi, "A lowprofile conical beam loop antenna with an electromagnetically coupled feed system," IEEE Trans. Antennas Propag., vol. 8, pp. 1864-1866, Dec. 2000.

[14] Jihak Jung, Wooyoung Choi, and Jaehoon Choi,"Small Wideband Microstrip-fed Monopole Antenna", IEEE Microwave and Wireless Components Letters, vol. 15, no.10, October 2005, pp.703-705.

[15] David m.Pozar and Susanne M.Voda,,A Rigorour(1987) Analysis of a Microstrip line fed patch antenna, IEEE Transaction on Antenna and Propagation), vol.35, no.12, ,pp. 1343-1350. 